\title{
Transient charging and discharging current study in pure PVF and PVF/PVDF fluoro polyblends for application in microelectronics
}

\author{
A K GUPTA*, R BAJPAI and J M KELLER \\ Department of Postgraduate Studies and Research in Physics \& Electronics, Rani Durgavati University, \\ Jabalpur 482 001, India
}

MS received 3 August 2007; revised 18 December 2010

\begin{abstract}
The transient current were analysed by considering the effect of variation of forming time, temperature, field and composition of blend specimens. Measurements indicated that transient charging and discharging currents exhibited thermally activated character but did not show mirror image behaviour at different temperatures and field values. The $\log I-\log t$ plots were found to follow the Curie-Von Schweidler law with the value of decay constant ' $n$ ' lying in the range of 0.029-2.9456. These observed characteristics also indicated that the transient charging in PVF:PVDF fluoro polyblends occur partly due to orientation of dipoles but predominantly due to trapped space charges and hopping of charge carriers amongst localized states. The modification in transient behaviour on blending PVDF with PVF have been explained on the basis of plasticization effect which increases free volume and molecular mobility and $T_{\mathrm{g}}$ modification in the trap structure.
\end{abstract}

Keywords. PVF; PVDF; polyblend; fluoropolymers; transient current.

\section{Introduction}

If we take a polymer and apply a step field to it, the field interacts with the bound and free charges causing current flow in the external circuit. Generally, this current, known as absorption or charging current, depends on the time elapsed after the application of the step field, usually it falls off at first and then becomes steady. After the step voltage has been removed, there is still current flowing in the external circuit, which is called, desorption or discharge current by Vandershueren and Linkens (1978). The discharge current is, in general, the mirror image of the charging current except that a steady-state current is not reached. The isothermal desorption current decays for a long time, depending upon the internal phenomenon taking place irrespective of the steady-state current level.

The analysis of the experimental conditions can lead to a quantitative as well as qualitative idea of the mechanisms at work reported by Natta et al (1965) and Mahendru (1977). Transient current measurements being tedious as being carried out over a long time and it is very useful, giving far more consistent results than the others. This being soon accounted of electrical and other perturbing influences affecting the discharge process far less than in other experiments involving fast decay processes.

\footnotetext{
*Author for correspondence (anandlug225@ rediffmail.com)
}

Despite numerous isothermal transient current measurements made and subjected to analytical studies, the subject of the origin of absorption and desorption currents is still afflicted by doubts and controversies. This is because more extensive studies on the effects of various parameters like field, temperature, electrode material are still desired. To make a step forward, i.e. to reach the goal of having a clear, irrefutable and concrete explanation for the mechanisms involved in the transient absorption and desorption current flow, the present work takes up the studies on miscible polyblend specimen of PVF and PVDF. Incidentally, fluoro polymeric materials are systems on which electrical transport studies have been practically neglected.

A careful screening of the literature available gives an idea about the extent to which work has been done in case of the two polymers polyvinyl fluoride (PVF) and polyvinylidene fluoride (PVDF), when they are taken together mostly as in isomorphic situation exposed by Natta et al (1969), Thielen (1994) and Maiman and Schacham (1994). Isomorphism is believed to be possible when the polymer chains (blend) or monomer units (co-polymer) are similar in conformation and size in the crystalline state. PVF and PVDF, which are vinyl fluoro polymers, have good weather resistance and are used for outdoor applications. PVDF is reported to be piezoelectric and also apparently ferroelectric in nature. Since PVDF is moldable, transducers are readily fabricated. The manner of blending the two polymers affects the resultant properties, like impact strength, hardness, toughness tensile strength and electrical breakdown strength etc. 


\section{Experimental}

\subsection{Material used}

For preparation of pure and polyblend samples, commercially available polymers of PVF (BDH, UK), with 126,000 $\bar{M}_{\mathrm{w}}$ and PVDF (Aldrich, USA) with $140,000 \bar{M}_{\mathrm{w}}$, in powder forms were supplied by M/S Redox, Jabalpur and were used without further purification. DMF was supplied by E-Merck Chemical Co. and were used as received due to their high purity (over $99+\%$ ).

\subsection{Thin film preparation}

The solution cast technique has been used to prepare pure and polyblend specimens of PVF and PVDF. The two polymers were dissolved in their common solvent dimethyl formamide (DMF), at a temperature of $60^{\circ} \mathrm{C}$ with constant stirring. A known quantity of the homogenous solution obtained was poured on a glass plate floating over mercury pool inside a microprocessor-controlled oven. The solvent was allowed to evaporate at $60^{\circ} \mathrm{C}$ over a period of $6 \mathrm{~h}$. Thus the specimens obtained were in the form of thin films of thickness \pm 25 micron (approx). For measurement of film thickness, digital micrometer gauge was used.

\section{Characterization}

\subsection{Transient current measurements}

For carrying out transient current measurements, the sample was sandwiched between electrodes of the measuring cell, which was mounted inside the oven. The desired temperature was obtained and the sample was kept at this temperature for 30 min to allow it to attain uniform temperature. Then a constant step voltage was applied and the charging current versus time characteristic was recorded. After one hour the field was removed and the discharging current versus time measurements were recorded. The currents were recorded using Keithley electrometer model 610C. For each measurement, a fresh sample was taken.

Discharging the sample produces a negative charge current. If the charge current (I0) in the equilibrium state is subtracted from the charging current, the charge and discharge current are identical for many dielectrics. Therefore, the transient current $I(t)$ as a function of time is obtained from both the charge and discharge current as given by the following relation (Takeishi and Mashimo 1982):

$$
\begin{aligned}
& I_{\text {charge }}(t)=I(T)+I_{0}, \\
& I_{\text {discharge }}(t)=-I(T) .
\end{aligned}
$$

\subsection{Differential scanning calorimeter (DSC)}

Due to unique capabilities for the evaluation of polymeric materials and blends, modulated DSC was used to detect the glass transition temperature $\left(T_{\mathrm{g}}\right)$. Modulated DSC (TA Instrument model 2910), Perkin Elmer, Singapore was used to characterize the glass transition temperature $\left(T_{\mathrm{g}}\right)$, in the temperature range from $-80^{\circ}$ to $40^{\circ} \mathrm{C}$. Liquid nitrogen was used to undergo the temperature up to $-80^{\circ} \mathrm{C}$ at the rate of $10^{\circ} \mathrm{C} / \mathrm{min}$. Differential scanning calorimeter (DSC) study gives the melting behaviour and the glass transition temperature $\left(T_{\mathrm{g}}\right)$. The thermal characterization using DSC also provides information regarding the blend morphology, the compatibility and miscibility of the two polymers, their crystallization etc.

\section{Results and discussion}

The origin of transient charging and discharging currents cannot be decided exactly, however, after a systematic analysis of the dependence of the current on various experimental parameters such as field strength, time and temperature, etc a tentative discrimination can definitely be made between the various mechanisms proposed for a given material. The general characteristic of the observed results are discussed below.

\subsection{Time dependence}

Figures 1 and 2 show variation of transient charging and discharging currents with time for pure PVF and PVF: PVDF polyblend having wt\% composition of 100:00, 90:10, $70: 30,50: 50$ charged at different temperatures of 40, 60, 80 and $100^{\circ} \mathrm{C}$ with fields 40 and $100 \mathrm{kV} / \mathrm{cm}$ over a period of time 15 to $10^{4} \mathrm{~s}$. The logarithmic plots are in general, linear and characterized by two slopes in most of the cases. Further, the transient charging and discharging current are, in general, mirror image of each other. The current decays at a faster rate for the first few seconds (short time region) and then decay rate slows down (long time region). The observed time dependence of the current transient may be represented by the Curie-Von Schweidler law 1907 (Von Schweidler 1907),

$$
I(t)=A(T) t^{-n},
$$

where, $t$ is the time after application or removal of the step field, $A(T)$ is a temperature dependent factor and $n$ the decay constant. The value of slope ' $n$ ' for the two regions i.e. $n_{1}$, for short time region and $n_{2}$ for long time region, have been summarized in tables 1-4. 


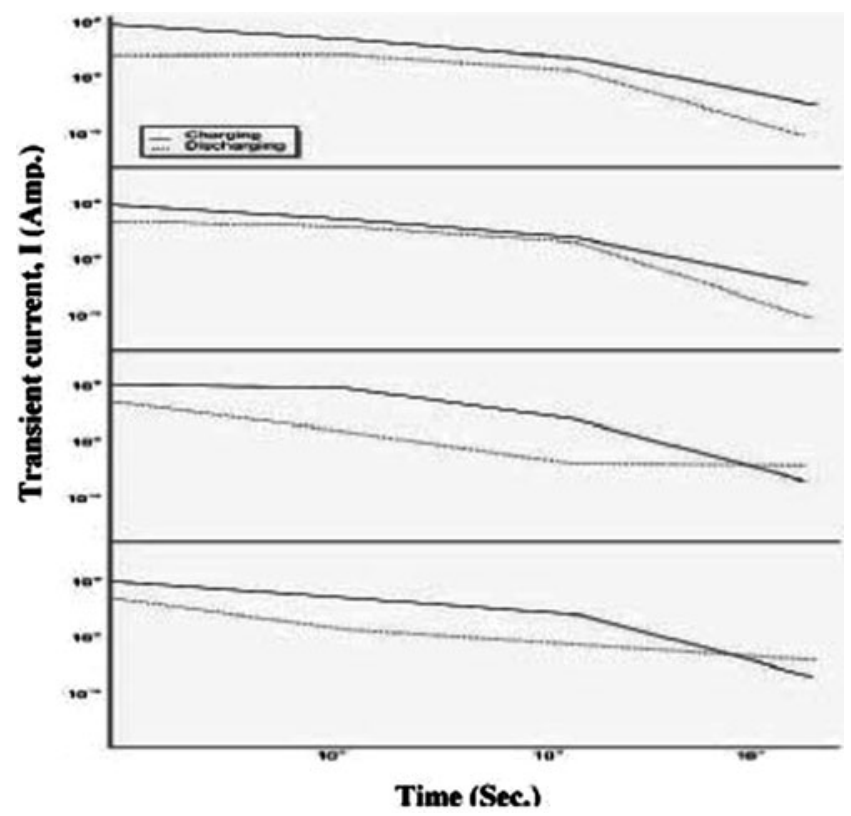

Figure 1. Time dependence of transient charging plot for pure PVF charge at different temperatures, i.e. $40,60,80$ and $100^{\circ} \mathrm{C}$ at $100 \mathrm{kV} / \mathrm{cm}$.

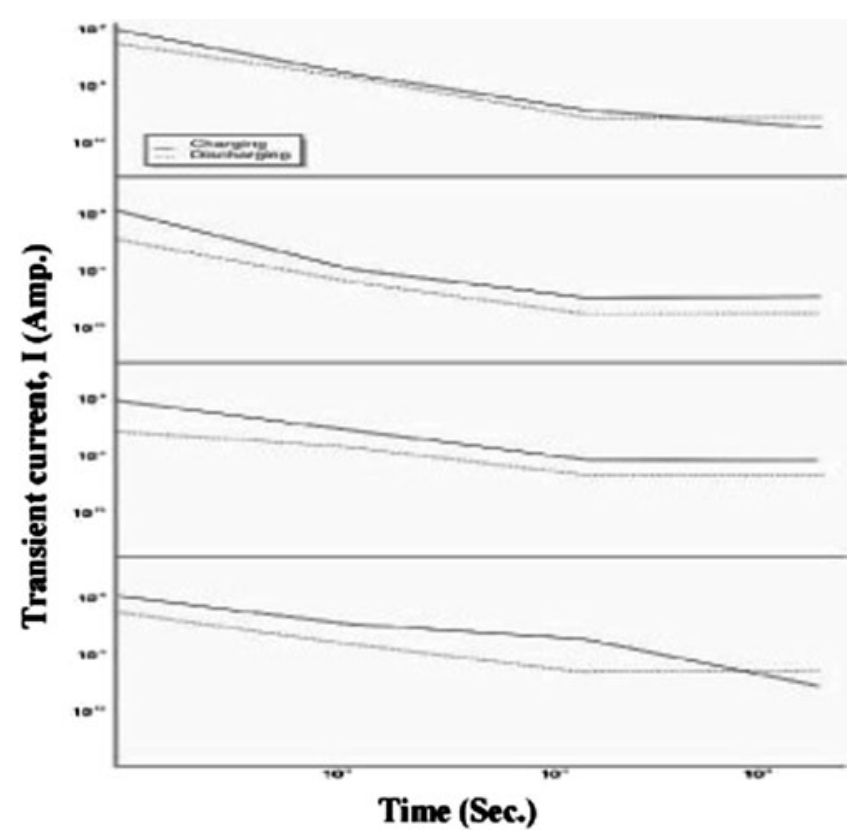

Figure 2. Time dependence of transient charging plot for PVF:50::PVDF:50 charge at different temperatures, i.e. 40, 60, 80 and $100^{\circ} \mathrm{C}$ at $100 \mathrm{kV} / \mathrm{cm}$.

\subsection{Temperature dependence}

The charging and discharging transient observed for the samples (pure PVF and PVF50:PVDF50 wt \%) charged with fields 40 and $100 \mathrm{kV} / \mathrm{cm}$ at different temperatures are shown in figures 3 and 4 . The curves exhibit $t^{-n}$ dependence. The charging and discharging currents are in general mirror images of each other. Further, the current departs processively from their mirror image nature at shorter times as temperature is increased. The temperature dependence of the observed transients can, however, be expressed more conveniently by plotting currents observed at various fixed times (isochronal) against temperature. Such isochronals have been constructed for various constant fields for fixed times 15, 30, 45 and $60 \mathrm{~s}$ (figure 5).

The isochronals are characterized by a peak located between 70 and $100^{\circ} \mathrm{C}$. A careful observation reveals that, the isochronal current profile tends to shift towards high temperature at shorter times. The transient currents thus exhibit the thermally activated behaviour (figure 6).

\subsection{Field dependence}

Figure 7 illustrates the variation of transient current with the forming field. Each figure corresponds to a particular constant charging temperature and wt $\%$ composition. From these plots it is clear that the dependence on field is not linear in nature.

\subsection{Composition dependence}

In the present investigation, the current measurements were made on pure PVF and PVF:PVDF polyblends having wt $\%$ composition of 100:00, 90:10, 70:30 and 50:50, respectively.

\subsection{Dielectric loss and Hamon's approximation}

As said earlier, the transient currents may be used to obtain information on the low frequency and also temperature dependence of its loss factor ' $\varepsilon$ '. For this purpose the discharge current data were converted from time-domain to frequency-domain using Hamon's (1995) approximation to give dielectric loss:

$$
\varepsilon^{\prime \prime}(f)=\frac{I(t)}{2 \pi f C_{\mathrm{a}} V} .
$$

The Hamon's frequency $(0 \cdot 1 / t$ and $V$ is independent of index $n$ in the range $1 \cdot 2 \geq n \geq 0 \cdot 2$.

Figure 8 shows the variation of dielectric loss, $\varepsilon^{\prime \prime}$, as a function of Hamon's frequency ( $\log \varepsilon^{\prime \prime}$ versus $\log f$ ) at constant temperature and charging fields for various blends. It can be observed from these curves that the dielectric loss decreases linearly with the increase in frequency in all cases.

Figure 9 illustrates the variation of dielectric loss as a function of temperature ( $\log \varepsilon^{\prime \prime}$ versus $T$ plots) at constant 
Table 1. Value of slope ' $n$ ' for the two regions, i.e. $n_{1}$ for shorter time region and $n_{2}$ for longer time region of pure PVF.

\begin{tabular}{|c|c|c|c|c|c|c|c|c|}
\hline \multirow{3}{*}{$\begin{array}{l}\text { Blend } \\
\begin{array}{l}\text { Field } \mathrm{kV} / \mathrm{cm} \\
\rightarrow \\
\downarrow \text { Temperature }\end{array}\end{array}$} & \multicolumn{8}{|c|}{ Pure PVF, 100:00 wt $\%$} \\
\hline & \multicolumn{4}{|c|}{ Charging current } & \multicolumn{4}{|c|}{ Discharging current } \\
\hline & \multicolumn{2}{|c|}{$80 \mathrm{kV} / \mathrm{cm}$} & \multicolumn{2}{|c|}{$100 \mathrm{kV} / \mathrm{cm}$} & \multicolumn{2}{|c|}{$80 \mathrm{kV} / \mathrm{cm}$} & \multicolumn{2}{|c|}{$100 \mathrm{kV} / \mathrm{cm}$} \\
\hline $100^{\circ} \mathrm{C}$ & $0 \cdot 486$ & $0 \cdot 1883$ & $0 \cdot 2023$ & 0.5668 & $0 \cdot 3874$ & 0.2936 & $0 \cdot 3033$ & 1.6222 \\
\hline $80^{\circ} \mathrm{C}$ & $0 \cdot 1257$ & $0 \cdot 1034$ & $0 \cdot 1286$ & $0 \cdot 3542$ & $0 \cdot 2484$ & 1.293 & $0 \cdot 3721$ & $0 \cdot 4528$ \\
\hline $60^{\circ} \mathrm{C}$ & $0 \cdot 2315$ & 0.4000 & 0.0984 & $0 \cdot 1692$ & 0.6894 & 0.023 & $1 \cdot 3528$ & $0 \cdot 0167$ \\
\hline $40^{\circ} \mathrm{C}$ & 0.4095 & 0.9991 & $0 \cdot 0246$ & $0 \cdot 1341$ & 0.0601 & - & $0 \cdot 5678$ & $0 \cdot 1524$ \\
\hline
\end{tabular}

Table 2. Value of slope ' $n$ ' for the two regions i.e. $n_{1}$ for shorter time region and $n_{2}$ for longer time region of PVF:PVDF::50:50 wt $\%$.

\begin{tabular}{|c|c|c|c|c|c|c|c|c|}
\hline \multirow{3}{*}{$\begin{array}{l}\text { Blend } \\
\text { Field kV/cm } \\
\downarrow \\
\downarrow \text { Temperature }\end{array}$} & \multicolumn{8}{|c|}{ PVF50:PVDF50 wt $\%$} \\
\hline & \multicolumn{4}{|c|}{ Charging current } & \multicolumn{4}{|c|}{ Discharging current } \\
\hline & \multicolumn{2}{|c|}{$80 \mathrm{kV} / \mathrm{cm}$} & \multicolumn{2}{|c|}{$100 \mathrm{kV} / \mathrm{cm}$} & \multicolumn{2}{|c|}{$80 \mathrm{kV} / \mathrm{cm}$} & \multicolumn{2}{|c|}{$100 \mathrm{kV} / \mathrm{cm}$} \\
\hline $100^{\circ} \mathrm{C}$ & 1.960 & $0 \cdot 240$ & 1.834 & $0 \cdot 2321$ & $0 \cdot 349$ & 0.094 & $0 \cdot 296$ & 0.052 \\
\hline $80^{\circ} \mathrm{C}$ & 1.789 & $0 \cdot 544$ & 1.674 & 0.4322 & 0.494 & $0 \cdot 1243$ & $0 \cdot 352$ & $0 \cdot 100$ \\
\hline $60^{\circ} \mathrm{C}$ & 0.9314 & $0 \cdot 3882$ & 0.659 & $0 \cdot 1243$ & $1 \cdot 000$ & $0 \cdot 3479$ & $0 \cdot 982$ & $0 \cdot 2988$ \\
\hline $40^{\circ} \mathrm{C}$ & 0.5616 & $1 \cdot 211$ & $0 \cdot 498$ & 0.9284 & $0 \cdot 146$ & 1.9432 & $0 \cdot 216$ & 1.7645 \\
\hline
\end{tabular}

Table 3. Value of slope ' $n$ ' for the two regions i.e. $n_{1}$ for shorter time region and $n_{2}$ for longer time region of PVF:PVDF::70:30 wt $\%$.

\begin{tabular}{|c|c|c|c|c|c|c|c|c|}
\hline \multirow{3}{*}{$\begin{array}{l}\text { Blend } \\
\text { Field kV/cm } \rightarrow \\
\downarrow \text { Temperature }\end{array}$} & \multicolumn{8}{|c|}{ PVF70:PVDF30 wt $\%$} \\
\hline & \multicolumn{4}{|c|}{ Charging current } & \multicolumn{4}{|c|}{ Discharging current } \\
\hline & \multicolumn{2}{|c|}{$80 \mathrm{kV} / \mathrm{cm}$} & \multicolumn{2}{|c|}{$100 \mathrm{kV} / \mathrm{cm}$} & \multicolumn{2}{|c|}{$80 \mathrm{kV} / \mathrm{cm}$} & \multicolumn{2}{|c|}{$100 \mathrm{kV} / \mathrm{cm}$} \\
\hline $100^{\circ} \mathrm{C}$ & 0.497 & $0 \cdot 2000$ & $0 \cdot 3458$ & $0 \cdot 325$ & $0 \cdot 3945$ & $2 \cdot 9456$ & 0.7342 & $0 \cdot 3000$ \\
\hline $80^{\circ} \mathrm{C}$ & $0 \cdot 2257$ & $0 \cdot 1234$ & $0 \cdot 2943$ & $0 \cdot 8992$ & $0 \cdot 2349$ & $0 \cdot 2941$ & 0.5696 & 1.0231 \\
\hline $60^{\circ} \mathrm{C}$ & $0 \cdot 3115$ & 0.4839 & $0 \cdot 1234$ & $0 \cdot 8656$ & $0 \cdot 6897$ & 1.000 & 0.4876 & 0.5635 \\
\hline $40^{\circ} \mathrm{C}$ & 0.4390 & $0 \cdot 0293$ & 0.0231 & $0 \cdot 2341$ & 0.891 & 0.4946 & $0 \cdot 3694$ & $0 \cdot 2415$ \\
\hline
\end{tabular}

Table 4. Value of slope ' $n$ ' for the two regions i.e. $n_{1}$ for shorter time region and $n_{2}$ for long time region of PVF:PVDF::90:10 wt\%.

\begin{tabular}{|c|c|c|c|c|c|c|c|c|}
\hline \multirow{3}{*}{$\begin{array}{l}\text { Blend } \\
\text { Field kV/cm } \rightarrow \\
\downarrow \text { Temperature }\end{array}$} & \multicolumn{8}{|c|}{ PVF90:PVDF10 wt $\%$} \\
\hline & \multicolumn{4}{|c|}{ Charging current } & \multicolumn{4}{|c|}{ Discharging current } \\
\hline & \multicolumn{2}{|c|}{$80 \mathrm{kV} / \mathrm{cm}$} & \multicolumn{2}{|c|}{$100 \mathrm{kV} / \mathrm{cm}$} & \multicolumn{2}{|c|}{$80 \mathrm{kV} / \mathrm{cm}$} & \multicolumn{2}{|c|}{$100 \mathrm{kV} / \mathrm{cm}$} \\
\hline $100^{\circ} \mathrm{C}$ & 0.4567 & 0.4555 & $0 \cdot 3495$ & $0 \cdot 8695$ & $0 \cdot 3133$ & 0.9888 & 0.5678 & $2 \cdot 641$ \\
\hline $80^{\circ} \mathrm{C}$ & $0 \cdot 2415$ & 0.0996 & $0 \cdot 2941$ & $0 \cdot 5674$ & $0 \cdot 2111$ & $0 \cdot 4567$ & $0 \cdot 3491$ & $1 \cdot 281$ \\
\hline $60^{\circ} \mathrm{C}$ & $0 \cdot 3197$ & 0.7411 & $0 \cdot 1564$ & $0 \cdot 3495$ & $0 \cdot 1497$ & $0 \cdot 3025$ & $0 \cdot 2561$ & $0 \cdot 841$ \\
\hline $40^{\circ} \mathrm{C}$ & 0.0988 & 0.5975 & $0 \cdot 1000$ & $0 \cdot 2000$ & $0 \cdot 3990$ & 0.6789 & 0.0988 & 0.782 \\
\hline
\end{tabular}




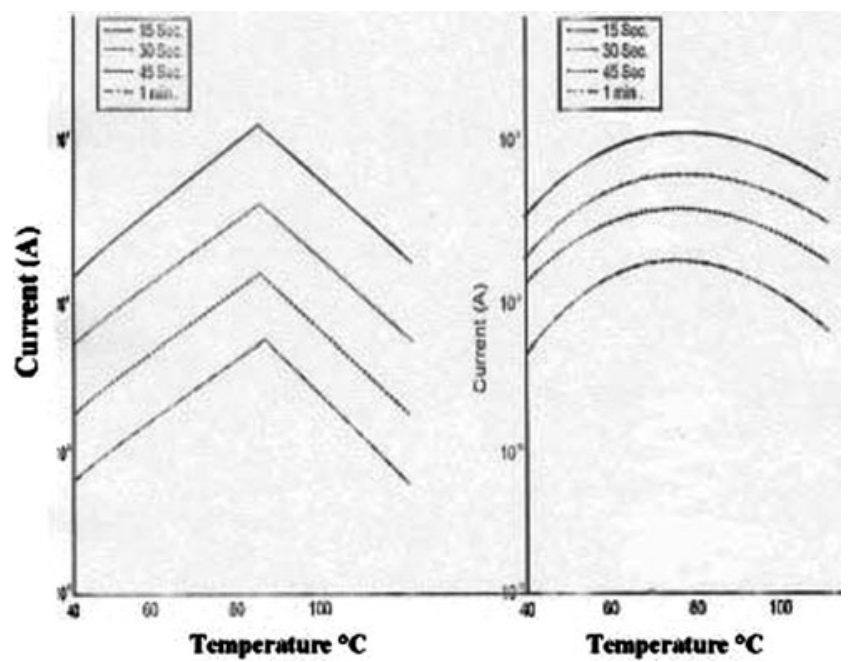

Figure 3. Temperature vs current plot for pure PVF with 40 and $80 \mathrm{kV} / \mathrm{cm}$ at different temperatures.

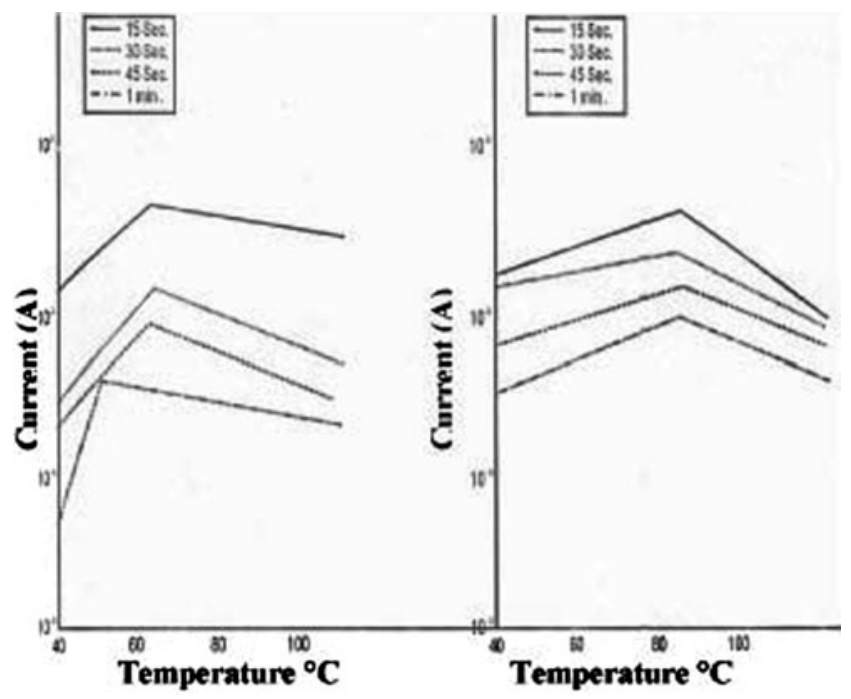

Figure 4. Temperature vs current plot for PVF:50::PVDF:50 with 49 and $80 \mathrm{kV} / \mathrm{cm}$ at different temperatures.

Hamon's frequency for various isomorphic polyblend specimen. The curves are characterized by dielectric loss maxima centred at about $80-100^{\circ} \mathrm{C}$. Further, from the nature of the curves, it is clearly evident that the profile of the curves shifts towards higher frequency with increasing temperature.

From the observed transient current characteristics it is evident that atleast two distinct mechanisms should be responsible for the current decay. One mechanism is operative in the range of the short times giving rise to a straight line on a $\log I$ vs $\log t$ plot with a particular value of decay constant $n_{1}$, and the other mechanism is operative in the range of long times giving rise to another straight line with a decay constant, $n_{2}$, of higher value.

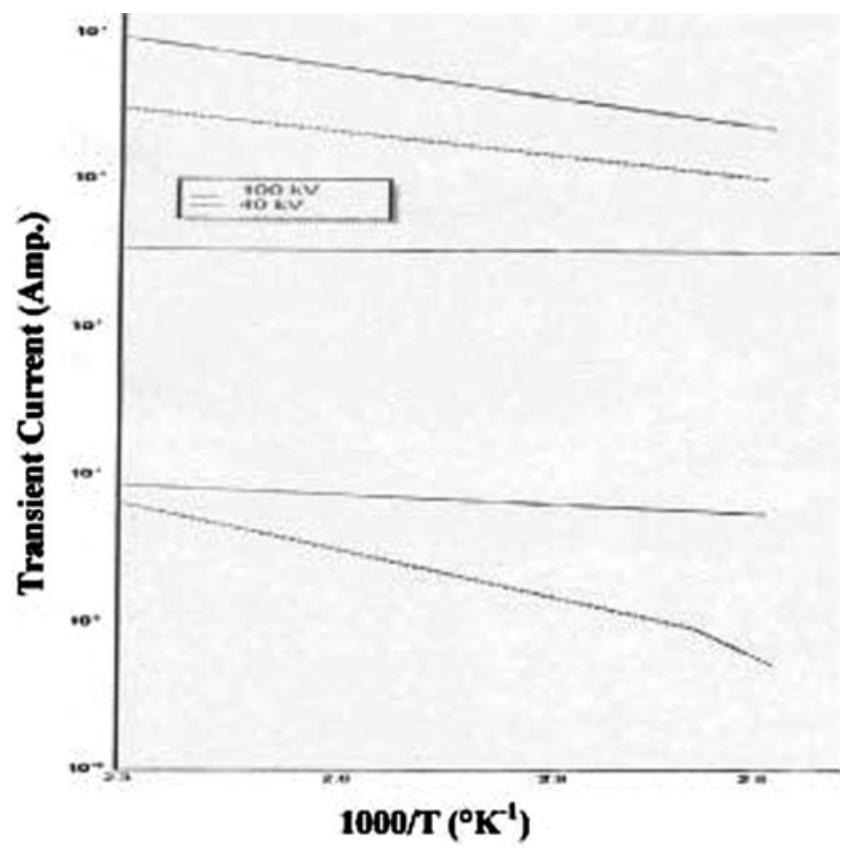

Figure 5. Plot of isochronal current vs $1000 / T\left({ }^{\circ} \mathrm{K}^{-1}\right)$ for PVF:50::PVDF:50 transient current at 40 and $100 \mathrm{kV} / \mathrm{cm}$.

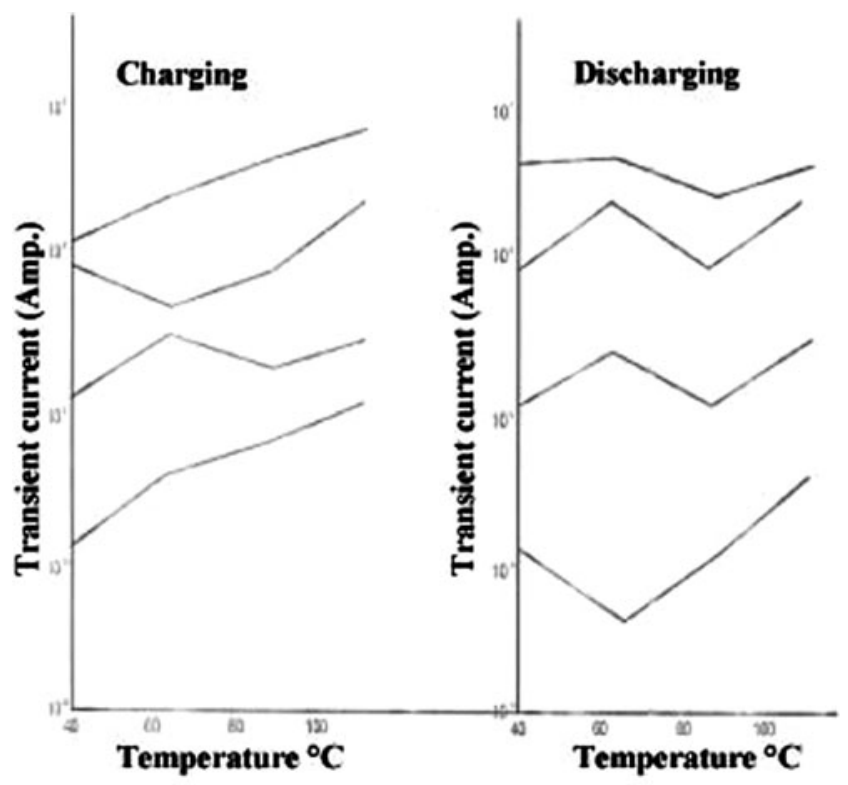

Figure 6. Isochronal current vs temperature plots of PVF:50:: PVDF:50 at $100 \mathrm{kV} / \mathrm{cm}$ at different temperatures, i.e. 40, 60, 80, $100^{\circ} \mathrm{C}$, curves $1,2,3$ and 4 represent $30,60,120$ and $240 \mathrm{~s}$, respectively.

The above results have been discussed in the light of the existing models, i.e. dipolar relaxation, charge trapping, charge injection leading to space charge effects and electrode polarization considering the variation of such currents with time, temperature, and blend composition. However, in the present case, charging and discharging transient at fixed 


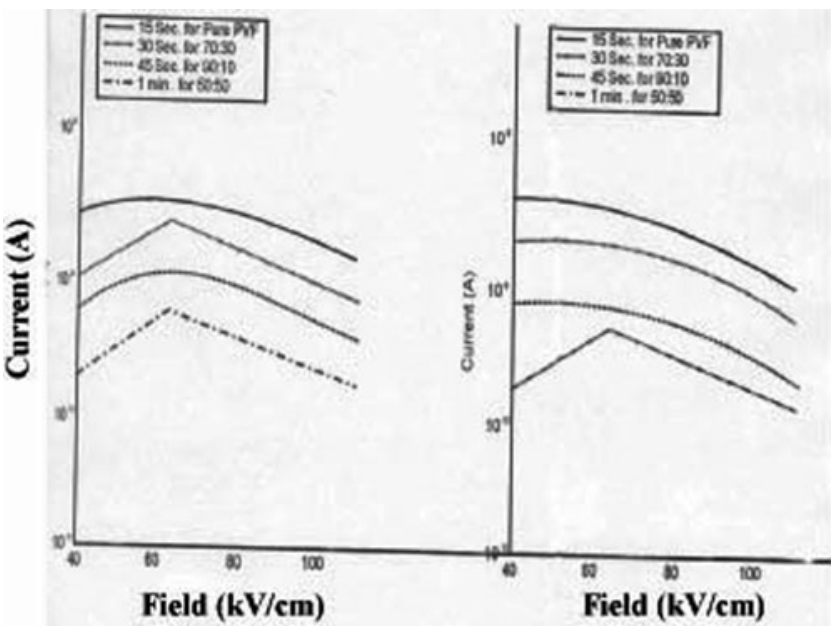

Figure 7. Variation of transient current for pure $\mathrm{PVF}$ and PVF:PVDF polyblend specimens recorded at $80^{\circ} \mathrm{C}$ and $100^{\circ} \mathrm{C}$ at various fields i.e. $40,60,80$ and $100 \mathrm{kV} / \mathrm{cm}$.

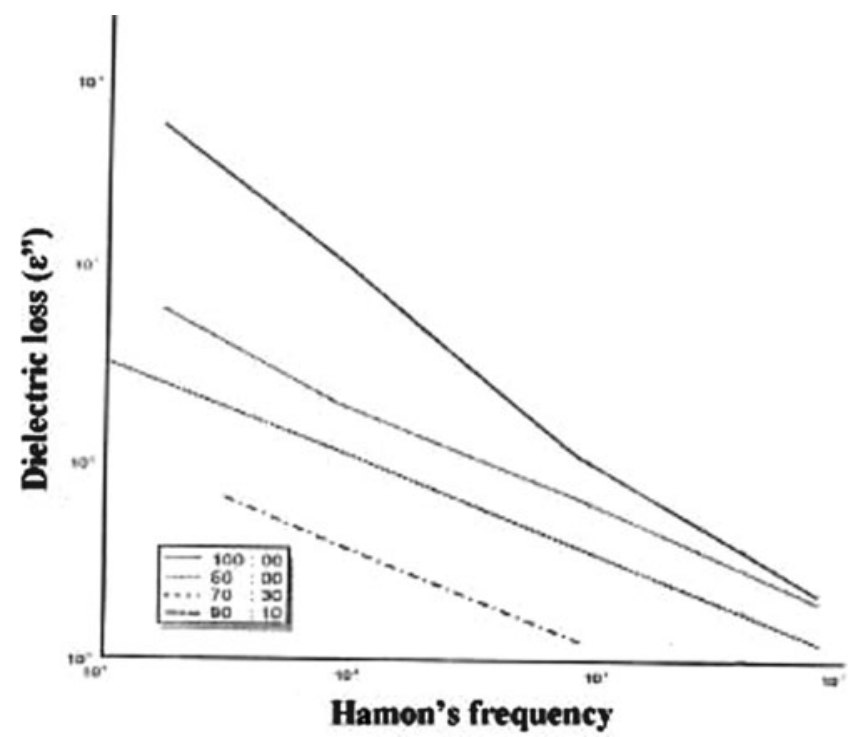

Figure 8. Dielectric loss dependence on Hamon's frequency log $\varepsilon^{\prime \prime}$ vs $\log f$ plot for pure PVF and PVF:PVDF polyblend charged with field of $100 \mathrm{kV} / \mathrm{cm}$ at charging temperature, $100^{\circ} \mathrm{C}$.

times show (isochronal) thermal dependence and exhibit a complex dependence on electric field. Thus it seems that tunneling of empty traps can be ruled out as a possible mechanism for the observed transients.

In case of electrode polarization, the transient currents are reported to be proportional to $t^{-n}$ with the value of the decay constant, $n=0.5$ at shorter times, and $n>1$ at longer times. However, in the present case, at shorter times the value of ' $n$ ' is found to vary from 0.029 to 0.5 and 0.03 to 1.3 at longer times; in case of charging and in case of discharging from 0.06 to 1.32 at shorter times and 0.016 to 2.94 at longer times. These observations show that the process of electrode polarization is unlikely to be dominant in the present case.

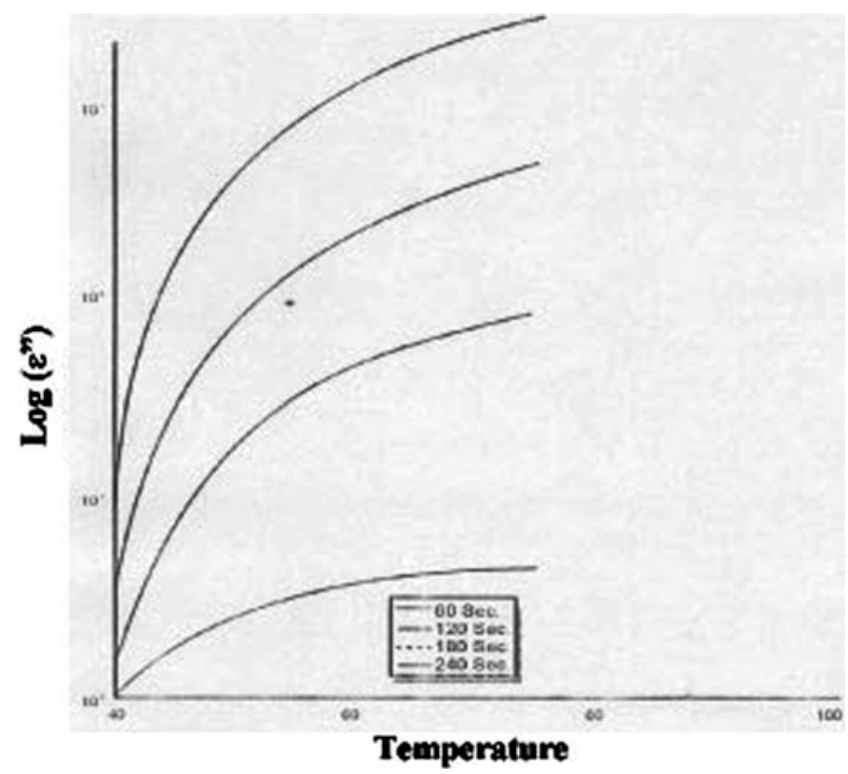

Figure 9. Temperature dependence on dielectric loss log vs temperature plot for PVF:PVDF polyblend charge with field of $100 \mathrm{kV} / \mathrm{cm}$.

Analysing the observed experimental result further, we know that dipolar relaxation can also account for $t^{-n}$ type of time dependence. However, it has to be borne in mind that, as a general rule, dipolar processes involved in polymers are characterized by a distribution in relaxation times, and that overlapping of several processes are likely to be present. This also implies that the Curie law can only be considered more or less a rough approximation of the real time dependence and can only depict the transient phenomenon over short periods of time.

Keeping this in mind following observed characteristics can be considered as evidence in favour of dipolar model from our experimental results:

(i) The isochronal current profile tends to shift towards higher temperature at shorter times, thus suggesting the presence of a thermally activated process.

(ii) Dielectric loss versus temperature curve at a particular constant frequency also exhibits characteristic similar to the isochronals.

(iii) The value of decay constant is less than two leaving few exceptions and can, therefore, be considered to be in the characteristic dipolar range of $0<n<2$.

Both PVF and PVDF are polar polymers. The $T_{\mathrm{g}}$ of PVF and PVDF are around -10 and -40 to $-50^{\circ} \mathrm{C}$ as shown in DSC thermograph (figures 10 and 11). The PVF is largely an amorphous polymer, while PVDF is semi-crystalline. The isochronal current profile and the observed peak may be attributed to relaxations due to micro-Brownian motions of the main chain in the amorphous phase. Thus, it can be concluded that the transient currents exhibit the expected behaviour of a dipolar relaxation. However, the relaxation is 


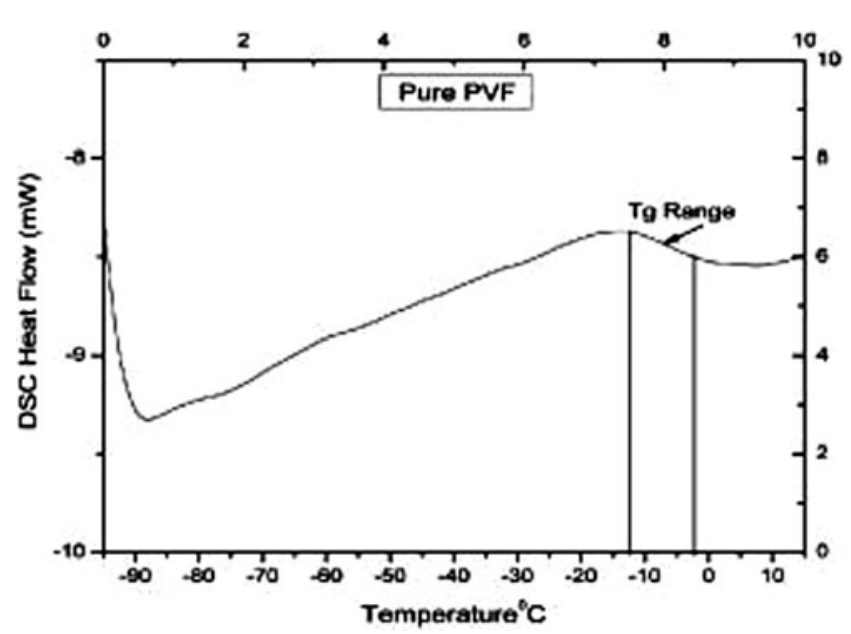

Figure 10. DSC thermograph of pure PVF.

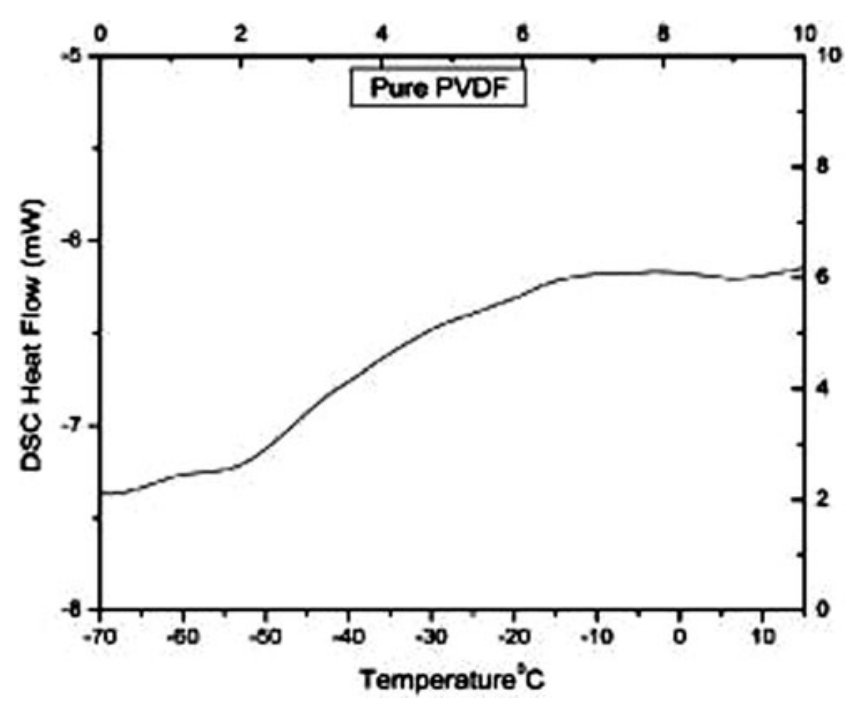

Figure 11. DSC thermograph of pure PVDF.

a WLF type process; the broadness of the isochronal peak and the profile suggests that there are several overlapping dipolar relaxations taking place in the amorphous region of the PVF:PVDF blend and contributing to this peak (figure 12).

The transient currents in time domain have actually been observed to follow the power law of the type, $I(t) \propto t^{-n}$; however, the logarithmic plot of the current are much steeper than the genuine characteristic (value of $n>1$ ). The observed current transient in the time-domain are due to dipolar mechanism, then the time domain response is made of two sequential decay processes. The decay at short times, i.e. at frequencies which are larger than the loss frequency $\omega_{\mathrm{p}}$, the current follows the law,

$$
I(t) \propto t^{-n}, \quad 0<n<1, \quad t \ll \frac{1}{\omega_{\mathrm{p}}} .
$$

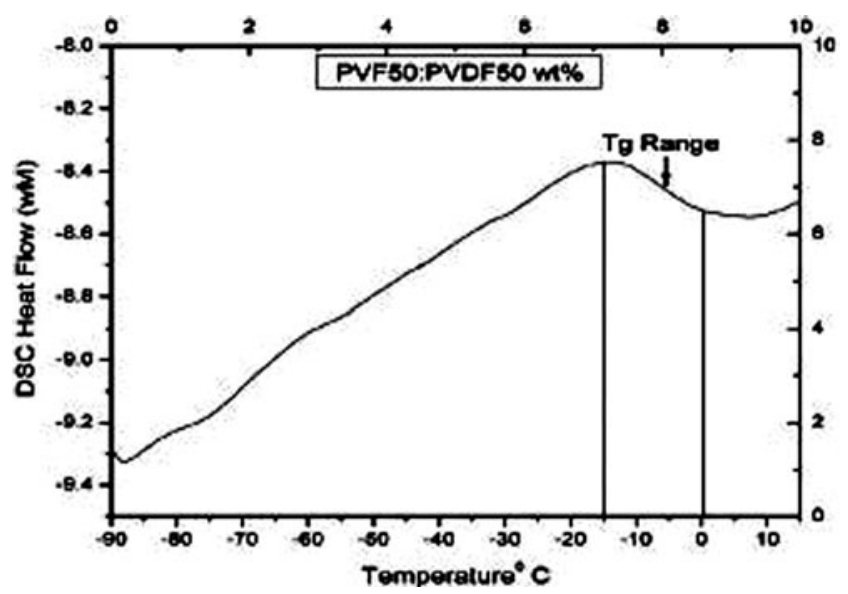

Figure 12. DSC thermograph of PVF50\%:PVDF50\% polyblend.

On the other hand, at larger times, the current obeys the relation

$$
I(t) \propto t^{1-m}, \quad 0<m<1, \quad t \gg \frac{1}{\omega_{\mathrm{p}}} .
$$

Thus, the two power laws determine the time domain response of a dipolar system in which a loss peak is seen in the frequency domain. If the system is carrier-dominated, the trapping of charge carriers takes place, i.e. electrons, ions or polarons become the dominant polarization species, then their low frequency dispersion below a frequency $\omega_{\mathrm{p}}$ which corresponds to long time range may be described by the same power law discussed above but with a very small value of constant $n$.

It may be advantageous, therefore, for the purpose of subsequent discussion to introduce the notation $n=1-p$, with $p$ close to unity to represent the low frequency dispersion region. In such a case the long time response of charge carrier system will be denoted by

$$
I(t) \propto t^{-1+p} ; p \approx 1, \quad t \gg \frac{1}{\omega_{\mathrm{p}}},
$$

which corresponds to a very slow time varying process. Thus, the time domain response covering both dipolar loss peaks and the strong low frequency dispersion associated with charge carrier dominated system may be represented in the unified form of the polarization current as a function of time,

$$
I(t) \propto t^{s}, \quad 0<s<1,
$$

with the exponent, taking values in different ranges at long and short times, respectively.

The observed values of decay constant $n$ (i.e. $n<2$ ) in most of the cases and the existence of two slopes indicate that the transient currents exhibit the expected behaviour of a dipolar relaxation mechanism.

In the present case the observed transient current varies nonlinearly with the applied field. Such behaviour cannot be 
explained on the basis of dipolar mechanism alone. With the aid of a two-side model for hopping mechanism, Guth and Mark (1934) and Mahendru et al (1976) have derived an expression for the transient current, which is general form of time-dependent current observed in the present case. In this model, there is no restriction on the value of $n$. The model assumes that the localized states are distributed in energy and charge carriers undergo limited transitions to associated adjacent sites. The observed nonlinear field dependence of the current and value of $n^{2}$ can be explained by hopping mechanism, which requires the existence of such localized states. PVF is amorphous in nature, whereas PVDF is semi crystalline. The existence of localized states in the bandgap is well documented in the case of amorphous system. Thus it can be concluded that hopping of charge carriers amongst the localized states may also contribute to the transient currents observed in the present case.

The existence of two values of exponent $n$ can be explained on the basis of the presence of shallow and deep traps. Trapping of charges can take place at various trapping sites-surface states, chain foldings and molecular disorder (Fisher and Rahl 1977; Murata and Koizumi 1989). The polymer blend is an heterogeneous system and as such it has a very large number of trapping sites. It appears that at shorter times and low charging temperatures, only shallow traps are involved which get implied earlier giving rise to higher value of $n$. At longer times and higher temperatures, more and more deep traps are filled. The trapping of charge carriers (electrons and holes) in deep traps may lead to induce dipole formation. The energy required for their disorientation and release of charge carriers from these trapping sites will be high and as such the discharge current and value of $n$ will be low.

Thus, in conclusion it may be suggested that the observed transient-current curves are composite in nature and can be explained on the basis of dipolar and space charge effects. The space charge polarization sets up with a fast setting time of $10^{-14}$ to $10^{-15} \mathrm{~s}$, while the dipolar polarization requires a longer time period because of inertia of dipoles and molecules. It appears that at shorter times, hopping charge and space charges contribute significantly resulting in a complex dependence on the charging field.

\section{Conclusions}

Measurements of time dependent charging and discharging currents also clearly point out that current transients in PVF and PVF:PVDF polyblends originate due to dipolar orientation and trapped space charge undergoing limited hopping amongst localized states. Further charging and discharging phenomenon depend strongly on the polymer structure.

\section{Acknowledgement}

Authors are thankful to UGC-DAE (consortium for scientific research), Indore, for providing DSC facilities.

\section{References}

Fisher P and Rahl P 1977 Prog. Colloid. Polym. Sci. 62149

Guth E and Mark H 1934 Monatsch. 6593

Hamon B V 1995 Proc. IEEE London 99151

Mahendru P C 1977 Phys. Status Solidi (a) 42403

Mahendru P C, Jain K and Mahendru P 1976 J. Phys. D Appl. Phys. 983

Maiman S and Schacham S E 1994 J. Appl. Phys. 754

Murata Y and Koizumi N 1989 IEEE Trans. Elect. Insul. 24449

Natta G, Allegra G, Bassi I W, Sianesi D, Poricco G and Tonfi G 1965 J. Polym. Sci. 34263

Natta G, Allegra G, Bassi I W, Sianrsi D, Carlion G, Chielini E and Montagholi G 1969 Macromol. 2311

Takeishi S and Mashimo S 1982 Rev. Sci. Instrum. 53115

Thielen A 1994 J. Appl. Phys. 758

Vandershueren J and Linkens A 1978 J. Appl. Phys. 494

Von Schweidler C 1907 Ann. Phys. LP3 24711 